\title{
Communication Skills Education in Advance Care Planning: a Survey Study in Medical Students at the University of Antwerp
}

Mick van de Wiel ( $\square$ mick.vandewiel@uza.be)

University Hospital Antwerp (UZA)

Katrien Bombeke

University of Antwerp

Annelies Janssens

University Hospital Antwerp (UZA)

\section{Research Article}

Keywords: Palliative Care, Advance Care Planning, Medical Education, Communication Skills

Posted Date: May 19th, 2021

DOI: https://doi.org/10.21203/rs.3.rs-477899/v1

License: (c) (i) This work is licensed under a Creative Commons Attribution 4.0 International License.

Read Full License 


\section{Abstract}

\section{Background}

Palliative Care (PC) medicine is strongly emerging worldwide. Despite the effort to implement PC in the basic medical curriculum in Belgium, little time is spent on it during theoretical training.

For two consecutive years, a survey was presented to all students from the second master's year medicine from the University of Antwerp. The survey consisted of demographic questions, a knowledge test about PC and an evaluation about students' self-confidence regarding communicative skills used in end-of-life conversations.

First, to evaluate students' self-confidence regarding end-of-life conversation and mapping out possible gaps in the palliative care training at our university. Second, to assess whether our classes are able to get students to adequately reflect on possible factors that may influence end-of-life conversation.

\section{Results}

Students feel that not enough time is spent on palliative care education. However, good scores were obtained regarding the theoretical knowledge about PC. Students feel confident about general communicative skills, but don't feel confident about communicative skills specific to palliative care/advance care planning.

\section{Conclusion}

Specific course content can help improve students' confidence in end-of-life conversation. No difference was seen between real-life teaching or online teaching. Regularly evaluating students' confidence can help alter course content for the future.

\section{Introduction/background}

Advance care planning (ACP) is an essential part of palliative care (PC). An exact definition for ACP is still lacking, but a consensus is formed. $(1,2)$ In general, ACP enables individuals at all stages of life to make plans about their future health care. The benefits of ACP are diverse. ACP can reduce unwanted hospitalisation, improve the quality of patient-clinician communication, increase the use of palliative care, and increase patient satisfaction and quality of life. $(3,4)$ The need for palliative care $(P C)$ is strongly emerging. Almost every physician trainee will come into contact with palliative patients during their internships. It is therefore important to include PC in the curriculum of medical students. Not only theoretical knowledge, but also the acquisition of communication skills is of great importance. In general, $P C$ is integrated into the fellowship and specialist training phase. The European Association of Palliative Care (EAPC) is actively working to include palliative care earlier in the curriculum of medical students. Despite the integration and obligation of the palliative care component during undergraduate years in many countries, including Belgium, the time amount spend on palliative care education is very limited.(5- 
9) With the scaling down of the medicine curriculum from 7 to 6 years, the subject "palliative care", which consisted of 1 week of knowledge sharing on palliative care, disappeared. As a result, an elective course on "palliative care" is offered to the students of the second master's degree. In addition, PC is briefly discussed in various disciplines (e.g. oncology, pneumology, neurology, etc.).

Each academic year, the medicine curriculum at the University of Antwerp integrates six 3-hour sessions on communication skills training. The communication lessons are situated in the CANMEDS role of "communicator".(10) With these lessons, we want to provide students with tools to improve their communication skills when they enter the practical training. Each lesson has its own theme: breaking bad news, active listening, shared decision making, conflict management, giving information, dealing with anxious or depressed patients, as well as a lesson on advance care planning.

The lesson on ACP has been structured as a "flipped classroom" lesson, in which students first watch a knowledge video on the topic We also ask the students to reflect on their own wishes and standards regarding ACP. In the first part, the video focuses deeper on specific subjects such as when is a patient palliative (surprise question), legal content surrounding euthanasia, declaration of intent and palliative sedation. In the second part, we work on specific communication skills in the plenary lesson. We want to provide tools to make various aspects of ACP discussions easier (e.g. discussing Do Not Resuscitate (DNR) code with the Allow Natural Death (AND)-method).(11) Lastly, we want to make sure that students are able to identify the tipping points that make it easier for doctors to start a conversation about ACP. This is an important issue since timing of an APC conversation remains challenging. In this way, situations that initially present themselves as barriers for a conversation can be transformed into a facilitating environment.(12)

In normal conditions, 8 teachers (for approximately 15 students each) give experiential sessions regarding communicative skills in palliative care. In 2020 however, the coronavirus-disease (covid) pandemic challenged our education system in many ways. Real-life classes were compulsorily converted to online classes. As a result, this lesson was given online for more than 100 medical students, by only 2 teachers. The lesson was given in plenary in combination with breakout rooms to give the students the opportunity to practice and make the session more interactive.

With this study, we wanted to determine whether medical students believe that they have acquired sufficient skills to work with palliative patients in practice. We tested the theoretical knowledge about PC and the self-confidence of students regarding specific communication skills on this topic. Secondly, we looked at whether the current way of teaching was able to improve student's specific communication skills on this topic. Thirdly, we took a look at how the corona pandemic caused a shift from live to online teaching and if this affected the training. Lastly, we wanted to know if we could succeed in getting students to understand that certain circumstances can be used to start a conversation about ACP. Therefore, we gauged students' opinions on how the corona pandemic might influence the conversation about ACP. With all this information we can map out possible gaps in the palliative care training and take them into account for further training. 


\section{Materials \& Methods}

Current Flemish education lasts six years and consists of a three-year bachelor's program and a threeyear master's program. In the bachelor's program, focus lies on normal organ function and physiology. In the master's program, focus lies on different pathologies and application of knowledge in clinical practice. After approval from the ethics committee, for two consecutive years (2019 and 2020), two cohorts of students from the second master's year from the University of Antwerp were recruited.

The survey consists of three parts. The first part contains demographic data of the participant. The second part contains four questions that assess the current knowledge about palliative care (scored on 11). As there is no standard questionnaire for palliative care knowledge, these questions were designed by our own teachers and according to the content of the palliative care lesson taught.(13)The third and final part consists of a Dutch translation of one domain of the Palliative Education Assessment Tool (PEAT). This tool comprises seven palliative care domains: palliative medicine, pain, neuropsychologic symptoms, other symptoms, ethics and law, patient/family/nonclinical caregiver perspectives on end-oflife-care, and clinical communication skills. The PEAT enables educators to describe a specific aspect of the curriculum and use the information for evaluation and educational reforming.(14)

After obtaining informed consent, the survey was completed a first time right before the lessons regarding communicative skills around advance care planning and a second time right after these lessons occurred. In the first cohort, the survey was manually distributed during another plenary lesson. The second round of the survey was then questioned online to the participants a few weeks later. In the second cohort, both parts of the survey were questioned online with a few weeks in between. The online survey program "LimeSurvey" was used to collect the online surveys. Data was exported to SPSS version 27. And SPSS version 27 was used for descriptive analysis.

In the second cohort we added a qualitative part to our survey. We asked students if they saw the Covid19 pandemic as a possible barrier or facilitator in engaging in end-of-life/advance care planning conversation. For this qualitative descriptive part of the study, the students' text responses were thematically analysed by two independent researchers using Excel. In a first phase, each researcher labeled the students' responses individually using open codes, close to the students' words. Notes and interpretations were added. In a next step, these open codes and text fragments were compared and categorized in overarching themes, while differences in interpretations where discussed in a joint session. Finally, an overview of the different themes was made.

\section{Results}

A total of 220 students participated in this study. Table 1 shows demographics of the questioned students and Fig. 1 shows the flow diagram of the participants. 
Table 1

Demographics $(n=220)$

\begin{tabular}{|ll|}
\hline Sex & \\
Fale & $87(40 \%)$ \\
Age & $133(60 \%)$ \\
Min-Max (years) & \\
Mean (years) & $20-47$ \\
Religion & 23 \\
None & \\
Catholic & $127(58 \%)$ \\
Islam & $79(36 \%)$ \\
Judaism & $8(3 \%)$ \\
Hinduism & $4(2 \%)$ \\
Other & $1(<1 \%)$ \\
Preferred Specialty & $1(<1 \%)$ \\
Family doctor medicine & \\
Specialist medicine & $64(29 \%)$ \\
No preference/idea at this moment & $9(4 \%)$ \\
\hline
\end{tabular}

Table 2.1 shows the percentage of students answering 'yes' on the following questions: "Do you think that enough time is spent on end-of-life-education?"; "Do you feel you have gained enough knowledge from the end-of-life classes?"; "Do you feel that you are able to choose the right moment in time and independently have a conversation with a patient about advance care planning?". In 2019 , about $60 \%$ of the students think that enough time is spent on end-of-life education. In 2020 , this is only true for about $50 \%$ of the students. In both years ( $82 \%$ for 2019 and $77 \%$ for 2020 ), a large proportion of students indicate that they have insufficient knowledge regarding palliative care. After the given lesson, the students feel that their knowledge has clearly improved, $67 \%$ and $70 \%$ indicate that they have acquired sufficient knowledge for 2019 and 2020 respectively. A minority of the students indicate that they are sufficiently able to independently conduct a conversation around advance care planning. (10\% in 2019 and $12 \%$ in 2020). After the lesson, this number increases significantly to $48 \%$ of students in 2019 and $40 \%$ of students in 2020 . 
Table 2.1

percentage of students who answered "yes" on these questions.

\begin{tabular}{|lllll|}
\hline & 2019 & & 2020 & \\
& T1 & T2 & T1 & T2 \\
& $(\mathbf{n = 8 3 )}$ & $(\mathbf{n = 6 3 )}$ & $(\mathbf{n = 1 3 7 )}$ & $(\mathbf{n = 1 1 5})$ \\
\hline Enough time spend on PC & $58 \%$ & $76 \%$ & $47 \%$ & $73 \%$ \\
\hline Enough knowledge about PC & $18 \%$ & $67 \%$ & $23 \%$ & $70 \%$ \\
\hline Independently conducting an ACP with a patient & $10 \%$ & $48 \%$ & $12 \%$ & $40 \%$ \\
\hline
\end{tabular}

Table 2.2

Knowledge test scores (scored on 11)

\begin{tabular}{|lllll|}
\hline Mean & 2019 & \multicolumn{3}{c|}{2020} \\
\cline { 2 - 5 } Median & T1 & T2 & T1 & T2 \\
& $(n=83)$ & $(n=63)$ & $(n=137)$ & $(n=115)$ \\
& 7,4 & 9,4 & 8,2 & 8,6 \\
\hline & 8 & 10 & 8 & 9 \\
\hline
\end{tabular}

Table 2.2 shows the average scores on part 2 of the survey (the knowledge test). In general, the students score well on these questions. The average scores before the lesson are lower than those after the lesson. 
Table 3

Self-reported confidence in the domains of communication skills concerning palliative care (PC) and advance care planning (ACP).

\begin{tabular}{|c|c|c|c|c|}
\hline \multirow[t]{2}{*}{ Domain } & \multicolumn{2}{|c|}{2019} & \multicolumn{2}{|l|}{2020} \\
\hline & $\begin{array}{l}T 1 \\
(n= \\
83)\end{array}$ & $\begin{array}{l}T 2 \\
(n= \\
62)\end{array}$ & $\begin{array}{l}\text { T1 (n } \\
= \\
135)\end{array}$ & $\begin{array}{l}\text { T2 (n } \\
= \\
110)\end{array}$ \\
\hline Able to cope with a palliative/dying patient & $23 \%$ & $61 \%$ & $24 \%$ & $62 \%$ \\
\hline Have respect for and knowledge of different cultures and beliefs & $95 \%$ & $97 \%$ & $85 \%$ & $92 \%$ \\
\hline Empathically engage in an interview/consultation & $98 \%$ & $98 \%$ & $95 \%$ & $98 \%$ \\
\hline $\begin{array}{l}\text { Able to select the relevant clinical information during the } \\
\text { interview with the patient and to convey it correctly }\end{array}$ & $59 \%$ & $65 \%$ & $53 \%$ & $65 \%$ \\
\hline Able to empathize with patient and/or family members & $99 \%$ & $98 \%$ & $99 \%$ & $99 \%$ \\
\hline $\begin{array}{l}\text { Able to cooperate and communicate well within a } \\
\text { multidisciplinary team }\end{array}$ & $71 \%$ & $87 \%$ & $78 \%$ & $93 \%$ \\
\hline $\begin{array}{l}\text { Negotiate treatment goals and communicate them to patient } \\
\text { and/or family members }\end{array}$ & $43 \%$ & $66 \%$ & $51 \%$ & $62 \%$ \\
\hline $\begin{array}{l}\text { Resolve conflicts or negotiate conflicts (e.g. around end-of-life } \\
\text { care) }\end{array}$ & $31 \%$ & $26 \%$ & $35 \%$ & $44 \%$ \\
\hline Have a bad news conversation & $70 \%$ & $68 \%$ & $71 \%$ & $70 \%$ \\
\hline Ask for informed consent & $77 \%$ & $92 \%$ & $84 \%$ & $87 \%$ \\
\hline $\begin{array}{l}\text { Discuss withholding of life-prolonging treatment (e.g. discuss } \\
\text { DNR code) }\end{array}$ & $35 \%$ & $53 \%$ & $30 \%$ & $54 \%$ \\
\hline $\begin{array}{l}\text { Discuss advance care planning (e.g. appoint a representative, } \\
\text { provide information on palliative care,...) }\end{array}$ & $22 \%$ & $71 \%$ & $14 \%$ & $60 \%$ \\
\hline $\begin{array}{l}\text { Discuss the impending death with the patient and/or family } \\
\text { members }\end{array}$ & $29 \%$ & $45 \%$ & $39 \%$ & $51 \%$ \\
\hline Report the death of a patient to family members & $29 \%$ & $45 \%$ & $44 \%$ & $50 \%$ \\
\hline
\end{tabular}

Table 3 shows the self-reported confidence of our students in different domains of communication skills concerning palliative care and advance care planning. Students questioned in 2019 report a high confidence on the topics: empathic attitude towards patients and/or family members, empathic attitude in conversations and respect for other cultures and beliefs. Students report a low confidence on the topics advance care planning, coping with a palliative patient and discussing the impending death with patient and/or family members. Students questioned in 2020 scored likewise results for the high confidence and a minor shift to the topic discuss withholding of life-prolonging treatment in the low confidence category. After the specific communication lessons in 2019, students score a higher confidence on most 
categories. The only topic scoring lower after the lessons is resolve conflicts or negotiate conflicts. In 2020 students score a higher confidence on all topics after the lessons.

Table 4.1

Students' answers on the question: What is the effect of the covid-19 pandemic on conversations around advance care planning.

\begin{tabular}{|lll|}
\hline $\begin{array}{l}\text { The covid-19 pandemic makes a conversation about advance care } \\
\text { planning... }\end{array}$ & $\begin{array}{l}\mathrm{T1}(\mathrm{n}= \\
\mathbf{1 3 8})\end{array}$ & $\begin{array}{l}\mathrm{T2}(\mathrm{n}= \\
\mathbf{1 1 6})\end{array}$ \\
\hline ... easier & $31 \%$ & $59 \%$ \\
\hline ... more difficult & $39 \%$ & $14 \%$ \\
\hline Arguments for and against & $8 \%$ & $14 \%$ \\
\hline There is no influence & $1 \%$ & $3 \%$ \\
\hline I don't know & $21 \%$ & $10 \%$ \\
\hline
\end{tabular}

Table 4.1 and 4.2 show the result of the descriptive part of the survey. Table 4.1 shows the raw extracted answers of the study question into 5 general categories. Before the lesson and the presentation of the article, the students' opinions were clearly divided into a "for" or "against". Most students (39\%) saw that the covid-19 pandemic could act as a barrier in engaging end-of-life conversations. On the contrary, a large group of students (31\%) felt that the pandemic could just act as a facilitator. A considerable amount of students (21\%) had no idea and a negligible amount of students $(1 \%)$ felt that there was no influence of the pandemic on engaging these conversations. Only a small amount of students (8\%) described both facilitating as limiting factors. After the lesson, the students' opinions were more nuanced. Most of the students (59\%) saw the pandemic as merely facilitating and a smaller amount of students (14\%) saw the pandemic as merely limiting the end-of-life conversation. More students on T2 (14\%) described both facilitating and limiting factors of the pandemic, compared to T1. 


\section{Cat. 1: pandemic as a facilitator}
A. Awareness $=$ confrontation with the disease in the environment or via media causes reflection
B. The pandemic as a starting point for discussion (by both doctor and patient)
C. Doctors get more experience with end-of-life conversations
D. Fear/emotion as a stimulus for engaging conversations
E. More attention for psychosocial well-being

Cat. 2: pandemic as a barrier

A. Verbal and non-verbal communication is more difficult (mouth masks, distance, online conversations)

B. Less contact with doctors

C. No presence of family at the meeting

D. Time/pressure of doctor/caregiver causing no room for conversations

E. Fear/emotion as a barrier (botch for conversation and for seeking contact with a doctor)

F. Polarization between population groups: society creates division and distrust

Cat. 3: no idea

Cat. 4: interpretation that advance care planning is a primarily logistical issue

Cat. 5: resistance to teaching: wishes to learn in practice, don't understand the question

Table 4.2 shows the more extensive responses, categorized in 5 main themes. Category 1 (pandemic as a facilitator) and category 2 (pandemic as a barrier) are further divided into 5 and 6 subcategories respectively. Overall, category 1 describes all factors that could contribute in engaging end-of-lifeconversations. The most frequently cited reason is that confrontation with the coronavirus causes people to reflect more frequently on their own desires for extended therapy. This confrontation happens both through the media and through people in the environment who fall ill. Another important reason is that the pandemic can be used as a starting point for end-of-life conversations. The potential hindering factors in category 2 are mainly described as being practical concerns: wearing a mouth mask, distance during conversations, online consultations rather than real-life consultations, organizing conversations,.... Another important category is that of delayed care due to regional measures. Of particular note is that students often indicate that there is a large factor of fear/emotion going on with the patient. This can both initiate and avoid the conversation around the end of life. Students' responses that state they had no idea of an influence between the pandemic and engaging end-of-life conversations were included in category 3. Category 4 includes all responses that consider advance care planning to be a purely 
logistical thing, namely: home nursing, home care, admissions to a palliative unit,.... In category 5 , we see the responses of students who believe that they cannot learn from a lesson or article, but that they have to learn in real life and the responses where the question was misinterpreted.

\section{Discussion}

The students participating in this study are only one step away from their internships and their immediate contact with possible palliative patients. At this stage, a large proportion of students indicate that there is not enough time spent on palliative care and advance care planning. At the University of Antwerp, very limited time is spend on this topic at the moment. Despite this fact, students have a good background medical knowledge around palliative care.

Our study shows that students score themselves overall as being confident in general communicative skills (e.g.. empathically engage in a conversation, have respect for different cultures and believes, ask for informed consent). However, when it comes to communicative skills specific to palliative care and advance care planning (e.g. able to cope with a palliative/dying patient, discuss advance care planning, negotiate treatment goals), they score themselves as being non-confident before the lesson and insufficient-confident after the lesson. These specific skills need to be intensively trained during the internships. Students may underestimate the power of good communication skills in clinical practice. Even in situations where own clinical expertise is lacking, good communication can help overcome insecurities and improve patient-doctor relationship.

We see from the results that after the specific lessons, the students feel more competent to start a conversation about advance care planning. A few topics remain difficult. First of all, when we look at the topic 'discussing DNR code', we see that this skill clearly improves after the lesson, but that there is still room for further improvement. Discussing a DNR-code is a very important issue in every hospital (and residential care facilities). Especially during the covid pandemic, it turned out that this is not obvious. It therefore seems to us to be a correct judgement of the students to label this skill as difficult. The difficulty of a DNR discussion is twofold. On the one hand, there is the medical aspect where underlying comorbidities play an important role in the possible decision to restrict therapy. On the other hand, the timing and how to initiate this conversation, is an important communication skill that is regularly violated in practice. It goes without saying that this is a skill that students will only develop over time and with practice. Communicating this message in the lesson itself can already be helpful for students. This does not alter the fact that this remains a topic on which further work is needed. To explore this further, we have created a new survey to explore how this impacts physicians and physician assistants in clinical practice. Secondly, students also indicate that they find it difficult to inform relatives that a patient has died or will die soon. In our opinion, this seems to be a situation that can be practised on.(15) Telling the family that a patient is dying or has died is an important task as a doctor. Every doctor should be able to do this. Because of this result, we decided to implement a new scenario during the lesson "breaking bad news" in which the student has to tell a simulation patient that a family member is shortly going to die. Lastly, resolving or negotiating conflicts is scored as a difficult topic. Here, too, we share the view that this 
remains a difficult topic in practice. During the lesson on diversity, we try to provide tools for doctors to deal with other cultures, religions, norms and values. There is also a specific lesson in which we work with a potentially angry or aggressive patient. Further experience in practice is needed to develop this skill.

Another important result is that we notice the same trend in confidence scoring when teaching in real life or teaching online. Therefore, in the context of student's self-confidence, online teaching forms a good alternative for the intense and focused group discussions. This could be of great importance as there are less teachers needed for teaching online compared to teaching these skills face-to-face in focused and limited groups. Nevertheless, for the effective learning of these communication skills, it seems appropriate to be able to teach these lessons in real-life.

The extra survey question regarding the covid-19 pandemic showed that students first of all think about the possible practical obstacles for end-of-life conversations (time constraints, little face-to-face contact, absence of family members, annoying aspect of mouth masks in both verbal and non-verbal communication, ...). After a reflective article as well as bringing it up in class, more students saw the possible benefits of the pandemic (self-reflection of patients, norms and values of individuals and families, the impossibility of receiving visitors during hospitalisation, ...). With this lesson, we initiated a more considerate attention in students to recognise challenging situations as a starting point for entering into end-of-life conversations.

As possible limitations for our study we note that in the first year of our research, response for the second survey was much lower than for the first survey. This could possibly imply a selection of students with specific interest in the topic palliative care. Also, this study only provides a picture of the situation at our own university.

\section{Conclusion}

A lot of students indicate that there is not enough time spent on palliative care and advance care planning. Despite this fact, theoretical knowledge regarding palliative care is sufficient. Our study also shows that students score themselves as being confident in general communicative skills, but lack confidence in specific communicative skills regarding palliative care and advance care planning. Confidence in these communication skills becomes stronger after specific lessons focusing on advance care planning and palliative care, but this remains challenging. As a result of this survey, some lessons in the subject "communicative skills" were adapted. This allowed us to provide students with new tools to address the gaps in their self-confidence. These specific skills must be trained intensively during the internship year. As an internship supervisor, it is therefore important to pay sufficient attention to engaging students in end-of-life conversations. We notice the same trend in this confidence scoring when teaching in real life or teaching online. We therefore state that the online way of teaching forms a good alternative for the focused group sessions.

\section{List Of Abbreviations}




\begin{tabular}{|ll|}
\hline ACP & Advance Care Planning \\
\hline AND & Allow Natural Death \\
\hline Covid & Coronavirus Disease \\
\hline DNR & Do Not Resuscitate \\
\hline EAPC & European Association of Palliative Care \\
\hline PC & Palliative Care \\
\hline PEAT & Palliative Education Assessment Tool \\
\hline
\end{tabular}

\section{Declarations}

\section{Ethics approval and consent to participate}

The survey was approved by the Ethical Committee of the University Hospital Antwerp (UZA). Informed consent from all participants was obtained. All methods were carried out in accordance with relevant guidelines and regulations

\section{Consent for publication}

Not applicable

\section{Availability of data and materials}

The datasets used and analysed during the current study are available from the corresponding author on reasonable request.

\section{Competing interests}

The authors declare that they have no competing interests.

\section{Funding}

No funding was obtained for this research

\section{Authors' contributions}

MvdW was responsible for the practical design and implementation of this study, as well as the preparation and analysis of the database. She was a major contributor in writing the manuscript. KB is responsible for the lessons regarding advance care planning and helped analyse and interpret the qualitative data. AJ was the supporting factor for distribution of the survey to the students and responsible for the lessons regarding advance care planning. All authors read and approved the final manuscript. 
Prof. Dr. Johan Wens helped with the review and design of our survey.

\section{References}

1. Sudore RL, Lum HD, You JJ, Hanson LC, Meier DE, Pantilat SZ, et al. Defining Advance Care Planning for Adults: A Consensus Definition From a Multidisciplinary Delphi Panel. J Pain Symptom Manage. 2017;53(5):821-32.e1.

2. Rietjens JAC, Sudore RL, Connolly M, van Delden JJ, Drickamer MA, Droger M, et al. Definition and recommendations for advance care planning: an international consensus supported by the European Association for Palliative Care. The Lancet Oncology. 2017;18(9):e543-e51.

3. Brinkman-Stoppelenburg A, Rietjens JA, van der Heide A. The effects of advance care planning on end-of-life care: a systematic review. Palliative medicine. 2014;28(8):1000-25.

4. Houben CHM, Spruit MA, Groenen MTJ, Wouters EFM, Janssen DJA. Efficacy of advance care planning: a systematic review and meta-analysis. Journal of the American Medical Directors Association. 2014;15(7):477-89.

5. Nakamura Y, Takamiya Y, Saito M, Kuroko K, Shiratsuchi T, Oshima K, et al. A survey of palliative medicine education in Japan's undergraduate medical curriculum. BMC Palliat Care. 2017;16(1):38.

6. Ilse B, Alt-Epping B, Kiesewetter I, Elsner F, Hildebrandt J, Laske A, et al. Undergraduate education in palliative medicine in Germany: a longitudinal perspective on curricular and infrastructural development. BMC medical education. 2015;15:151.

7. Starmer D, Jamrozik K, Barton M, Miles S. Evaluating curriculum changes in undergraduate cancer education. Journal of cancer education : the official journal of the American Association for Cancer Education. 2004;19(3):156-60.

8. Downar J. Resources for Educating, Training, and Mentoring All Physicians Providing Palliative Care. Journal of palliative medicine. 2018;21(S1):S57-s62.

9. Carrasco JM, Lynch TJ, Garralda E, Woitha K, Elsner F, Filbet M, et al. Palliative Care Medical Education in European Universities: A Descriptive Study and Numerical Scoring System Proposal for Assessing Educational Development. J Pain Symptom Manage. 2015;50(4):516-23.e2.

10. Neville A WW, Martin D, Samson L, Feldman P, Wallace G, Jamoulle O, Francois J, Lussier M-T, Dojeiji S. Communicator. In: Frank JR, Snell L, Sherbino J, editors. CanMEDS 2015 Physician Competency Framework Ottawa: Royal College of Physicians and Surgeons of Canada. 2015.

11. Levin TT, Coyle N. A communication training perspective on AND versus DNR directives. Palliative \& supportive care. 2015;13(2):385-7.

12. van de Wiel M, Bombeke $K$, Wens J, Vanclooster P, Mertens V, Raskin J, et al. The Covid-19 pandemic could facilitate an end-of-life conversation. TvGG. 2020;77(3). 
13. Frey RA, Gott M, Neil H. Instruments used to measure the effectiveness of palliative care education initiatives at the undergraduate level: a critical literature review. BMJ supportive \& palliative care. 2013;3(1):114-9.

14. Meekin SA, Klein JE, Fleischman AR, Fins JJ. Development of a palliative education assessment tool for medical student education. Academic medicine : journal of the Association of American Medical Colleges. 2000;75(10):986-92.

15. Lewis C, Reid J, McLernon Z, Ingham R, Traynor M. The impact of a simulated intervention on attitudes of undergraduate nursing and medical students towards end of life care provision. BMC Palliat Care. 2016;15:67.

\section{Figures}

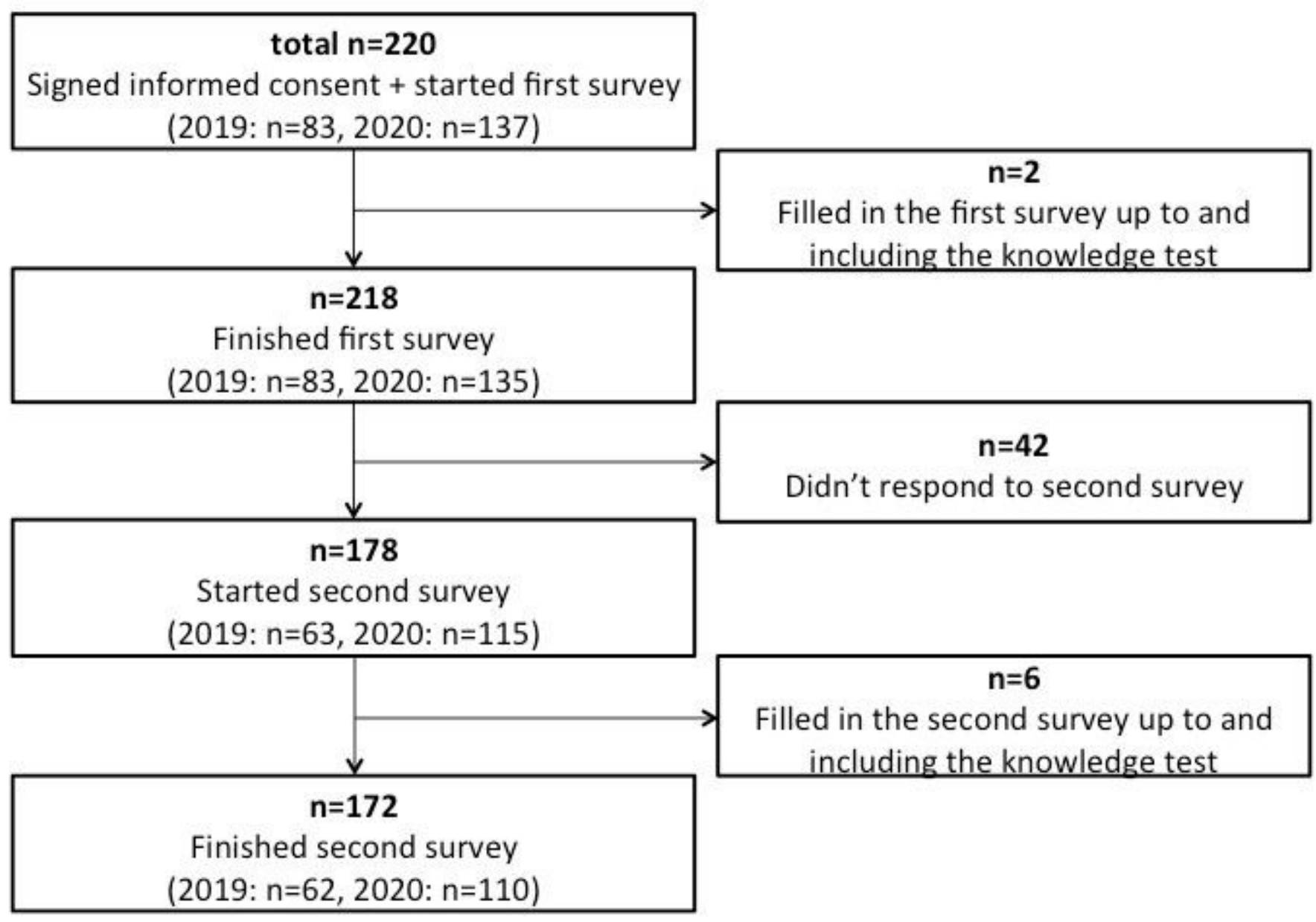

Figure 1

Flow diagram 